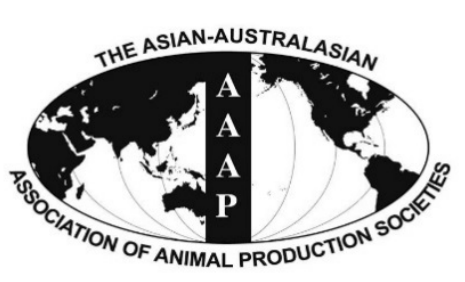

Open Access

Asian Australas. J. Anim. Sci.

Vol. 29, No. 2 : 299-306 February 2016

http://dx.doi.org/10.5713/ajas.15.0353

www.ajas.info

pISSN 1011-2367 elSSN 1976-5517

\title{
Body Temperature Monitoring Using Subcutaneously Implanted Thermo-loggers from Holstein Steers
}

\author{
Y. Lee ${ }^{1, a}$, J. D. Bok ${ }^{1, a}$, H. J. Lee ${ }^{1}$, H. G. Lee ${ }^{2}$, D. Kim ${ }^{1,3}$, I. Lee ${ }^{1,3}$, S. K. Kang ${ }^{1, *}$, and Y. J. Choi ${ }^{1, *}$ \\ ${ }^{1}$ Institute of Green-Bio Science and Technology, Seoul National University, Pyeongchang 25354, Korea
}

\begin{abstract}
Body temperature (BT) monitoring in cattle could be used to early detect fever from infectious disease or physiological events. Various ways to measure BT have been applied at different locations on cattle including rectum, reticulum, milk, subcutis and ear canal. In other to evaluate the temperature stability and reliability of subcutaneous temperature (ST) in highly fluctuating field conditions for continuous BT monitoring, long term ST profiles were collected and analyzed from cattle in autumn/winter and summer season by surgically implanted thermo-logger devices. Purposes of this study were to assess ST in the field condition as a reference BT and to determine any location effect of implantation on ST profile. In results, ST profile in cattle showed a clear circadian rhythm with daily lowest at 05:00 to 07:00 AM and highest around midnight and rather stable temperature readings (mean \pm standard deviation [SD], $37.1^{\circ} \mathrm{C}$ to $37.36^{\circ} \mathrm{C} \pm 0.91^{\circ} \mathrm{C}$ to $1.02^{\circ} \mathrm{C}$ ). STs are $1.39^{\circ} \mathrm{C}$ to $1.65^{\circ} \mathrm{C}$ lower than the rectal temperature and sometimes showed an irregular temperature drop below the normal physiologic one: $19.4 \%$ or $36.4 \%$ of 54,192 readings were below $36.5^{\circ} \mathrm{C}$ or $37^{\circ} \mathrm{C}$, respectively. Thus, for BT monitoring purposes in a fever-alarming-system, a correction algorithm is necessary to remove the influences of ambient temperature and animal resting behavior especially in winter time. One way to do this is simply discard outlier readings below $36.5^{\circ} \mathrm{C}$ or $37^{\circ} \mathrm{C}$ resulting in a much improved mean $\pm \mathrm{SD}$ of $37.6^{\circ} \mathrm{C} \pm 0.64^{\circ} \mathrm{C}$ or $37.8^{\circ} \mathrm{C} \pm 0.55^{\circ} \mathrm{C}$, respectively. For location the upper scapula region seems the most reliable and convenient site for implantation of a thermo-sensor tag in terms of relatively low influence by ambient temperature and easy insertion compared to lower scapula or lateral neck. (Key Words: Subcutaneous Temperature, Cattle, Fever Detection, Thermo-logger, Circadian Rhythm)
\end{abstract}

\section{INTRODUCTION}

Recently, frequent epidemic outbreaks in domestic animals such as foot and mouth disease (FMD) and avian influenza (AI) seriously threaten the national economy in many countries (Joo et al., 2002; Olsen et al., 2005; McLaws et al., 2009). For instance, approximately 3.48 million of cattle and pigs were buried in South Korea

\footnotetext{
* Corresponding Authors: S. K. Kang. Tel: +82-33-339-5731, Fax: +82-33-339-5732, E-mail: kangsk01@snu.ac.kr / Y. J. Choi. Tel: +82-2-880-4807, Fax: +82-2-875-7340, E-mail: cyjcow@ snu.ac.kr

2 Department of Animal Science and Technology, Konkuk University, Seoul 05029, Korea.

${ }^{3}$ College of Veterinary Medicine, Seoul National University, Seoul 08826, Korea.

a These authors contributed equally to this work.

Submitted Apr. 23, 2015; Revised May 6, 2015; Accepted Jun. 11, 2015
}

during the FMD outbreak from 2010 to 2011 consequently causing a property loss over 3 billion dollars (Park et al., 2013). To prevent such massive economic damage from pandemics in domestic animals, an early detection system should be set in place before disease outbreaks occur to reduce the time of detection. In a case study of a simulated outbreak of FMD in California, $\$ 565$ million of additional economic loss was forecast for every hour delay of detection after 21 days emphasizing the critical importance of early detection methods including body temperature (BT) monitoring systems which could alert farm workers to deal properly and promptly with the situation (Carpenter et al., 2011).

In homeotherms, fever is a complex physiologic reaction to maintain homeostasis and is also important defense mechanism against infection and inflammation. Thus, elevation of body temperature could be one of the primary diagnostic symptoms for clinical viral or bacterial 
infections (Dalal and Zhukovsky, 2006) and a most useful measurable indicator of many economically important events in cattle such as calving and estrus (Bewley, 2006). Thus body heat monitoring is central for early detection of infectious disease as well as cattle physiological events. Various ways to measure BT have been applied at different locations of cattle including rectum, reticulum, milk, and ear canal. Merits and limitations have been noticed for each different location and measuring methods. For continuous BT monitoring in cattle, reticulum, milk and tympanic canal has been tried as major sites and several systems are commercialized (Prendiville et al., 2002; Bewley et al., 2008; Adams et al., 2013; TekVet Health Monitoring System by TekVet Technologies Co.; TempTrack by DVM Systems, LLC.; TempVerified by FeverTags LLC). In-line milk temperature monitoring with a milk meter is one way to detect BT and mastitis, but it is only applicable to milking cows. A temperature sensing reticulum bolus system also has limitations such as short communication distance between bolus and a reader, battery problems, high price (\$40/bolus) and high fluctuations depending on the feed and water temperature. For example, cows receiving cold water showed a large drop of $9.2^{\circ} \mathrm{C}$ which did not come back to baseline temperature for a $3 \mathrm{~h}$ period (Bewley and Schutz, 2010). Temperature sensing with an ear canal radiotelemetry system is a promising one in terms of long distance wireless communication ( $\sim 90 \mathrm{~m}$ ) suitable for large cattle farm and rather simple attachment method the same as ear tagging. However this method also has shortcomings such as temperature stability, falloff of tagged devices and system cost. Another competing location has been under the skin (Brown et al., 1977; Bitman et al., 1984; Cilia et al., 1998; Hutu et al., 2009). Various subcutaneous temperature (ST) monitoring systems have been commercialized and used in laboratory conditions and research purposes, but they also suffered from short communication distance (few $\mathrm{cm}$ ) and strong influences (up to $9^{\circ} \mathrm{C}$ lower in the very cold temperature) from ambient temperature (Brown et al., 1977; Thermo-transponder IPPT-100 or -300 by BMD System).

Cattle hide is covered with well-developed hair, so temperature stability may differ depending on the implanting site. However, long term ST monitoring data from cattle is not available yet in field settings throughout the seasons.

Presently, this research group is performing a project to develop a real time BT monitoring system for early detection of infectious disease in domestic cattle by combining a Radio Frequency Identification (RFID) thermo-sensor tag which would be subcutaneously implanted into each cattle (Won et al., 2012). These BT monitoring systems are designed to detect a cow with abnormally elevated body temperature, immediately alert a farm manager who will check and take the necessary action such as isolation of the suspicious cow from the herd to prevent further spread of a pathogenic infection.

In this study, in order to evaluate the temperature stability and reliability of ST in highly fluctuating field conditions, long term ST readings were collected and analyzed from cattle in autumn/winter and summer season using commercial thermo-loggers surgically implanted in three different locations.

\section{MATERIALS AND METHODS}

\section{Animals}

The animal experiment was approved by IACUC (Institutional Animal Care and Use Committee) of Seoul National University and all applicable national laws and University policies regarding the care and use of animals were observed during the experiment. Seven Holstein steers (5 month-old) were used in this study. During the experiment, steers were housed together in a free stall barn without any heating system which was enclosed with solid fences and roof. The steers were given concentrate feed according to manufacturer's beef cattle rearing program (Cargill Agri Purina, Inc., Sungnam, Korea). Rice straw, mineral blocks, and water were fed ad libitum.

\section{Temperature measurement}

Rectal temperature was measured using a digital body thermometer MT-16C2 (Microlife AG, Widnau, Switzerland) in summer and winter time. In summer (in July), rectal temperature was measured during day time, 10 times a day at 1 hour intervals for 3 consecutive days, from eight Holstein calves (under 3 month-old) and in winter (in February), three times a day, at 10:30 AM, 1:30 PM, and 4:30 PM, for 4 consecutive days, from eight Holstein steers aged from 3 to 21 month-old. Subcutaneous body temperature was measured using button-shaped digital thermo-loggers (iButton DS1922L, Maxim Integrated, San Jose, CA, USA), which were preset to measure the body temperature at 1 hour intervals and surgically implanted into three different sites around neck area of steers during autumn to winter season (07 September 2013 to 22 February 2014) and summer (01 June 2014 to 30 September 2014) (Figure 1).

\section{Thermo-logger implantation and recovery}

Seven Holstein steers were sedated by intramuscular injection of $2 \%$ xylazine $(0.1 \mathrm{mg} / \mathrm{kg})$, properly restrained, hair-clipped, and locally anesthetized with $10 \mathrm{~mL}$ of $2 \%$ lidocaine around three implantation areas (lateral neck, upper, and lower scapula). A 2 to $3 \mathrm{~cm}$ long incision was made in the skin and undermined to make subcutaneous pocket, thermo-loggers inserted and then sutured with a medical stapler. Sedation was stopped immediately by 
A.

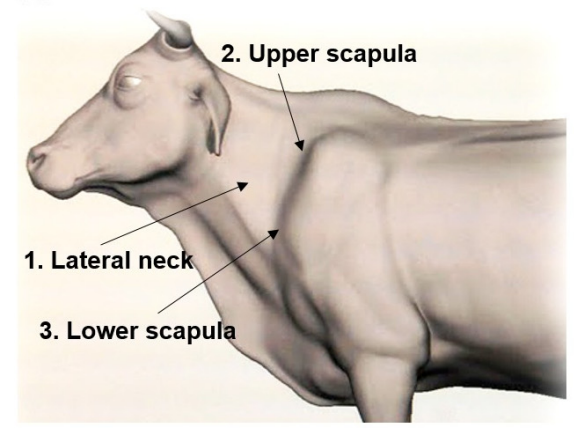

B.

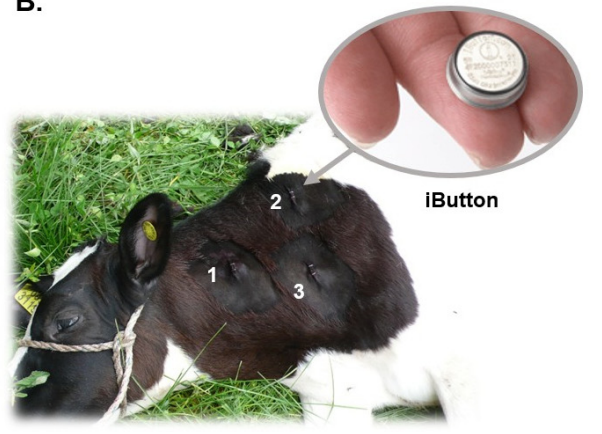

Figure 1. The implanting sites of thermo-loggers to monitor and assess the subcutaneous body temperature stability and reliability. (A) Schematic location of thermos-logger implantation, (B) Picture showing the thermos-logger (iButton) and insertional location.

injecting intravenously $0.5 \mathrm{~mL}$ of Atipamezole. After 4 to 6 months, the thermo-loggers were recovered by surgery following the procedure above. Data were computationally retrieved from the thermo-loggers and analyzed using OneWireViewer program (Maxim Integrated Products).

\section{Statistical analysis}

SPSS ver. 21 program (IBM Co., Armonk, NY, USA) was used for all the statistical analysis. The mean of body temperature from three implantation sites (lateral neck, lower and upper scapula) and at distinct time in the rectum were compared by on-way analysis of variance and Tukey's procedure using SPSS ver. 21 program $(\mathrm{p}<0.05)$. To determine the circadian rhythm of subcutaneous body temperatures of Holsteins, the observation value was collected from every one hour per month. This was analyzed descriptively to determine measure of variability of subcutaneous temperature for three implantation sites using box-and-whisker plot using Prizm 6 program (GraphPad Software, Inc., La Jolla, CA, USA) for Window. Values of more than 1.5 interquartile ranges above the $75 \%$ quartile or 1.5 interquartile ranges below the $25 \%$-quartile was considered as outliers in the box-and-whisker plots.

\section{RESULTS AND DISCUSSION}

\section{Body temperature test modalities}

Body temperature of animals could be measured by wide variety of methods and at different locations such as rectal thermometry, implantable microchip temperature transponder, an environmental noncontact infrared thermometer, wireless reticular boluses or ear canal temperature sensor and a panel reader located at a parlor entrance or exit, or a tympanic infrared thermometer. Rectal temperature is mostly regarded as a reference of core body temperature. So a close agreement between the test modality and the rectal temperature is required for a reliable temperature monitoring method (Cilia et al., 1998; Goodwin, 1998; Bewley et al., 2008; Adams et al., 2013; Thermo-transponder IPPT 300 by BMD System; TekVet
Health Monitoring System by TekVet Technologies Co.; TempTrack by DVM Systems, LLC.; TempVerified by FeverTags LLC). Temperature differences among the test modalities were generally small and stable in these studies due to the stable ambient temperature (Cilia et al., 1998; Goodwin, 1998). Authenticity of body temperature will depend on the accuracy of test modality and the insulation of body temperature from the ambient influences. Rectum is well protected by thick muscle and animal tail, and has ample blood circulation. Other body parts such as ear canal and reticulum also well insulated. Skin is well covered by hair met, but it is rather thin (3 to $5 \mathrm{~mm}$ thickness except hair) and rather short of blood circulation, thus easily affected by outside temperature fluctuations, especially in very low temperature (Brown et al., 1977).

Commercially available implantable thermotransponders are very reliable and easy way to measure the subcutaneous body temperature due to the small size of microchip transponder and easy-to-use implanter system (Thermo-transponder IPPT 300 by BMD System). But the communication distance between a transponder and a reader is very short $(<5 \mathrm{~cm})$ and temperature readings occur only when a reader transmits energy and signal to the transponder. Thus its use is rather limited in temperature controlled laboratory conditions and for short term measurement. In cattle barn conditions, the ambient temperature are highly fluctuating and animals are very active. So continuous monitoring of subcutaneous temperature means regular scanning of a reader to the cattle skin very near the implant site which is hard to achieve in field situation. In this study, small button shaped thermoslogger (iButton) was selected for the long battery life and high logging memory suitable for the implantation and long term ST data logging. Without any sealing capsule, this thermo-logger showed water-resistant property at least in atmospheric condition (data not shown). Manufacturers' temperature setting of each button was very accurate showing less than $0.1^{\circ} \mathrm{C}$ variance among the loggers. 

Profiling of the subcutaneous temperature from
Holstein steers

A RFID thermo-sensor tag implanted under the hide together with appropriate radiotelemetry data retrieval system could be one of competing BT monitoring systems such as reticulum bolus, ear canal thermometry, and in-line milk meter for early detection of infectious disease such as mastitis, pneumonia and other epidemic infectious disease in cattle (Brown et al., 1977; Hutu et al., 2009; Won et al., 2012). But long term stability and reliability data for ST in cattle are not available. Thus, long term subcutaneous temperature data of total 54,192 readings were obtained and analyzed in cattle. Digital thermo-loggers were surgically implanted to each steer in three different sites (lateral neck, upper scapula, and lower scapula) (Figure 1), then recovered after 6 months of autumn to winter or 4 months of summer season. The accumulated ST data were analyzed in comparison with barn (environmental) temperature (Figure 2).

As shown in Figure 2, the barn temperature declined continuously during experimental period representing seasonal temperature change, from autumn to winter, and ST profiles showed daily circadian rhythm representing temperature difference between day and night (Figures 2 to 4). This kind of circadian temperature up and down is typical, lowest in early morning (05:00 to 07:00 AM) and highest around midnight measured in ear canal, udder, or peritoneal cavity (Bitman et al., 1984;
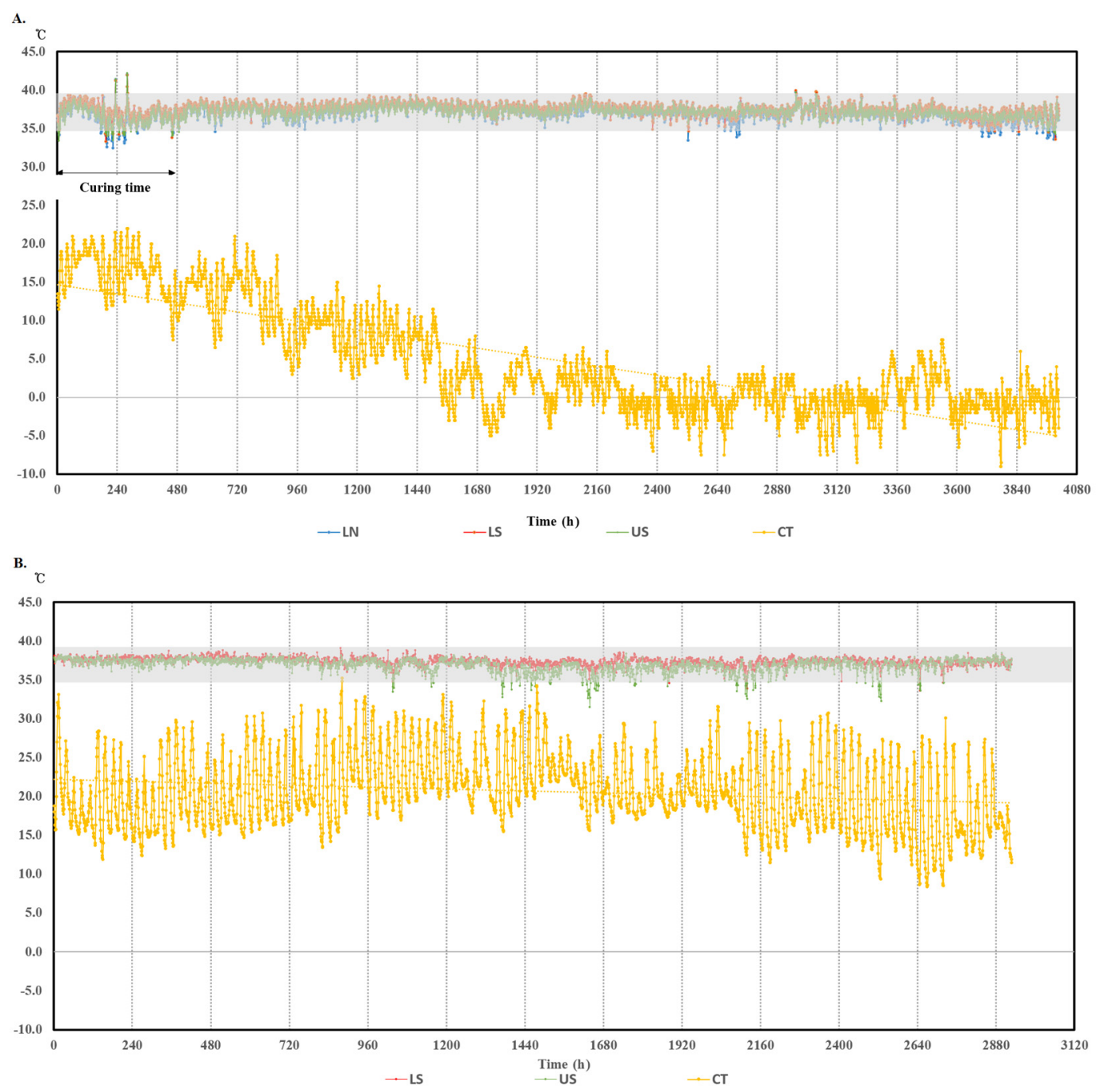

Figure 2. The long-term profile of bovine subcutaneous temperature collected in field. Body temperatures were measured by subcutaneously implanted thermo-logger in Holstein steers (blue, lateral neck; red, lower scapula; green, upper scapula; orange, cow shed temperature). Time (h) means cumulative temperature logging hour starting from the devise implantation. Temperature readings are rather fluctuating for the first 480 hours as the insertional wounds healed. (A) Winter experiment starts 07 September 2013 and ends 22 February 2014 and (B) summer experiment starts 01 June 2014 and ends 30 September 2014. Temperature inside the cow shed frequently dropped to subzero from 11 November 2013, but the mean and range values of subcutaneous body temperature remained stable with no significant difference compared to warmer days. LN, lateral neck; LS, lower scapula; US, upper scapula; CT, cow shed temperature. 
A. $\mathbf{L N}$

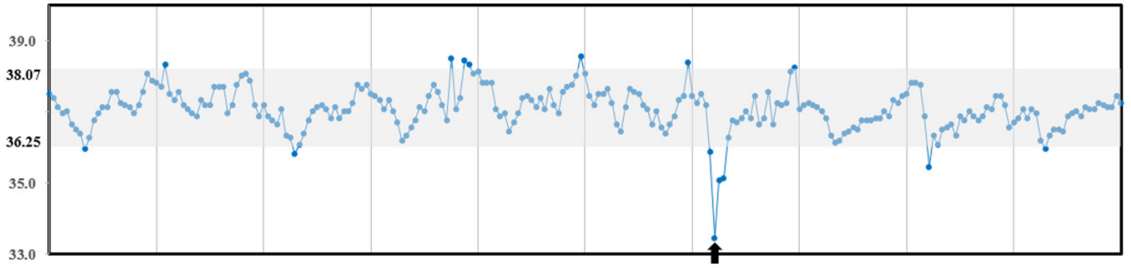

B. Us

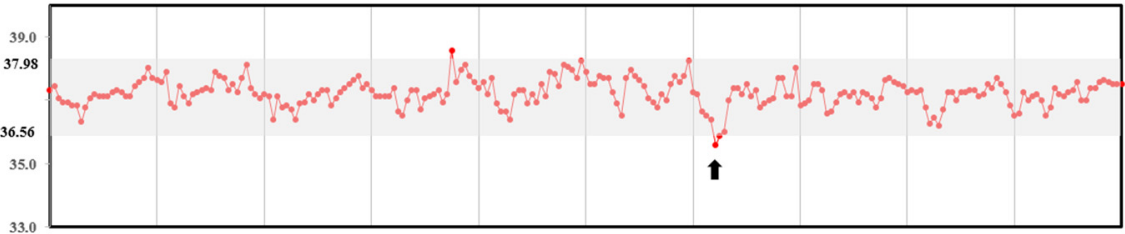

C.LS

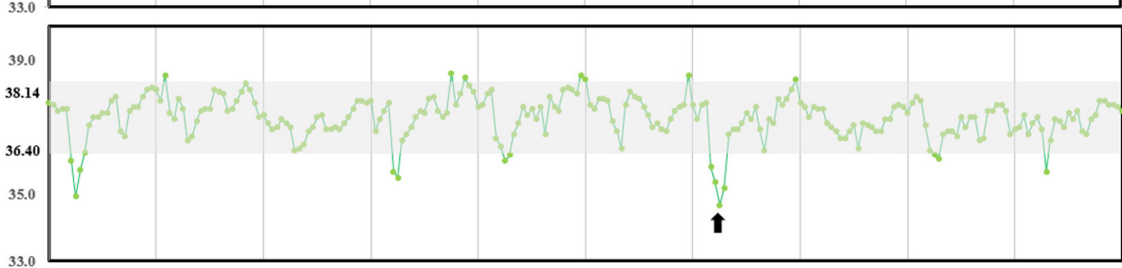

D.CT

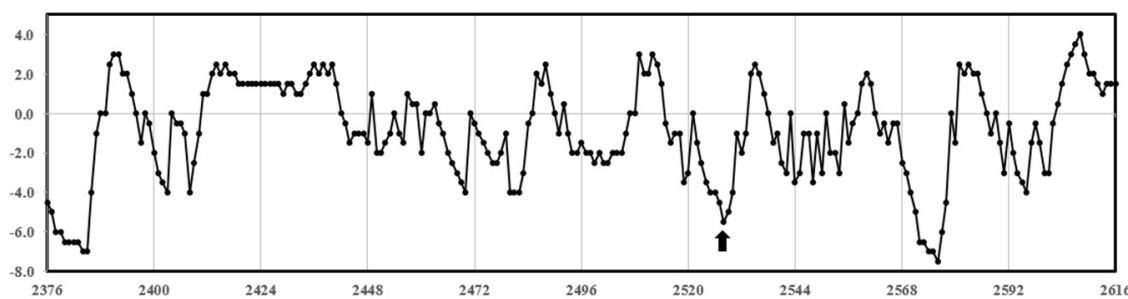

Figure 3. Representative daily pattern of subcutaneous body and cow shed temperatures for 10 days. (A) LN (lateral neck), (B) US (upper scapula), (C) LS (lower scapula), and (D) CT (cow shed temperature). Graph starts December 15 and ends December $25,2013$. Arrows indicate the temperature dip matching with the lowest shed temperature every early morning. Shaded areas are indicating the temperature ranges calculated as mean \pm standard deviation. Temperatures were recorded 1 hour interval and vertical line in the graph indicates one day. Overall subcutaneous body temperature profiles showed daily circadian rhythm coinciding roughly with shed temperature.

http://www.fevertags.com/\#!cattle-fever/c1n8o). The overall subcutaneous temperature profiles revealed steady maintenance even at sub-zero environmental temperature during experimental period. The temperature ranges at subcutaneous (54,192 readings collected for about 10 month period) and rectal areas are shown in Table 1 . The rectal temperature $\left(39.29^{\circ} \mathrm{C} \pm 0.33^{\circ} \mathrm{C}\right.$ ) of young calves (3month old) in summer was $1^{\circ} \mathrm{C}$ higher compared to that $\left(38.20^{\circ} \mathrm{C} \pm 0.27^{\circ} \mathrm{C}\right.$ ) of cattle (5 to 21 -month old) measured in winter. This difference may come from age and/or ambient temperature difference. However, the subcutaneous temperatures measured in three different locations were lower in mean values $\left(1.39^{\circ} \mathrm{C}\right.$ to $\left.1.65^{\circ} \mathrm{C}\right)$ and ranged more variable in standard deviation (standard deviation [SD], $0.91{ }^{\circ} \mathrm{C}$ to $1.02^{\circ} \mathrm{C}$ ) compared with the rectal ones. From these results, it is indicated that ST is affected by environmental influences. On the other hand, in Figure 2a, two high temperature peaks above $40^{\circ} \mathrm{C}$ are clearly seen

Table 1. Body temperature ranges of subcutaneous and rectal areas of Holstein steers

\begin{tabular}{lccccccc}
\hline Site & $\mathrm{N}$ & $\mathrm{Mean} \pm \mathrm{SD}$ & $\mathrm{SE}$ of mean & Minimum/maximum & Median & 25\%-Quartile/75\%-quartile & Quartile range \\
\hline Lateral neck & 17,040 & $37.16^{\mathrm{a}} \pm 1.02$ & 0.007 & $31.9 / 42.7$ & 37.3 & $36.6 / 37.9$ & 1.3 \\
Lower scapula & 21,456 & $37.36^{\mathrm{b}} \pm 0.91$ & 0.007 & $31.5 / 42.0$ & 37.4 & $36.8 / 37.9$ & 1.1 \\
Upper scapula & 15,696 & $37.10^{\mathrm{c}} \pm 0.91$ & 0.007 & $31.6 / 42.8$ & 37.2 & $36.6 / 37.7$ & 1.1 \\
Rectum $^{1}$ & & & & & & & \\
$\quad$ Winter & 354 & $38.20^{\mathrm{d}} \pm 0.27$ & 0.015 & $37.2 / 38.9$ & 38.2 & $38.0 / 38.4$ & 0.4 \\
$\quad$ Summer & 224 & $39.29^{\mathrm{e}} \pm 0.33$ & 0.022 & $37.5 / 40.3$ & 39.3 & $39.1 / 39.5$ & 0.4 \\
\hline
\end{tabular}

SD, standard deviation; SE, standard error.

${ }^{1}$ Rectal temperature at winter season was measured three times a day from 8 Holstein steers of 3 to 21 -month old, yielding 354 readings, and at summer measured ten times a day in one hour interval for 3 days from 8 Holstein calves, yielding 224 readings.

${ }^{a-e}$ Mean with different letters are significantly different $(p<0.001)$. 
around $240 \mathrm{~h}$ which indicate infection. These peaks are in 10 days in winter season which was cropped from Figure 2a. the curing period from surgical insertion of thermos-loggers. In this graph, it could be recognized the existence of daily Figure 3 shows three subcutaneous (lateral neck, upper and circadian rhythm in subcutaneous temperature profiles of all lower scapula) and cow shed temperature (CT) profiles for three insertional positions, with similar pattern, but

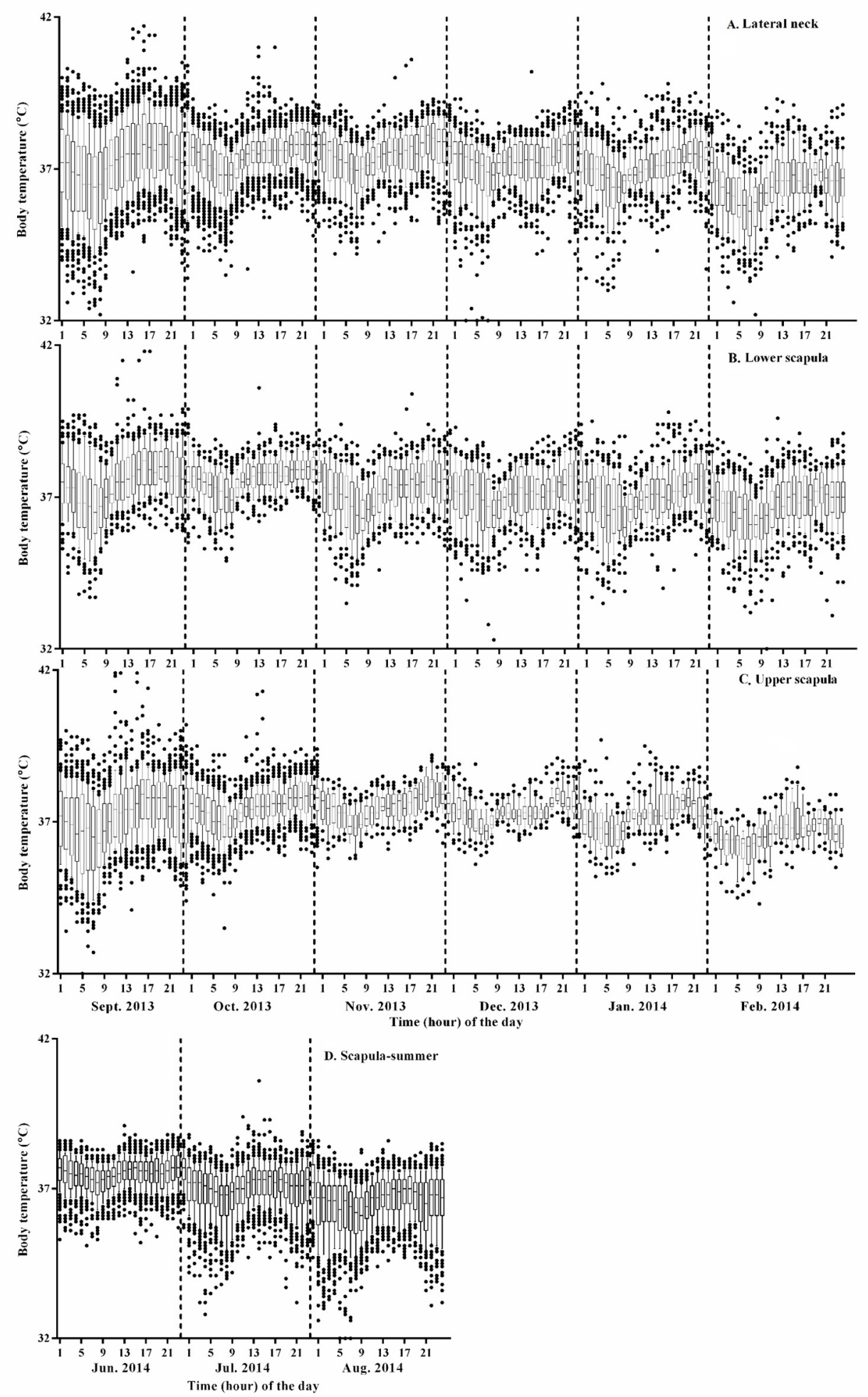

Figure 4. The cumulative presentation of monthly temperature reading. Monthly readings at each insertion sites were combined for each hour of the day and monthly temperature profiles were graphed. Circadian rhythmic fluctuations are clear in this monthly combined graph with apparent outliers below physiological temperature ranges. Over $40^{\circ} \mathrm{C}$ readings found in middle of September column are indicating the fever. Labels: (A) lateral neck, (B) lower scapula, (C) upper scapula, and (D) lower and upper scapula at summer. 
relatively small amplitude compared with environmental temperature profiles. To graphically analyze the stability and reliability of STs in different season, all the temperature readings of each month were put together in 24-h graph showing clear circadian rhythm (Figure 4). There were many outlier temperature readings under $36^{\circ} \mathrm{C}$ (as low as $31{ }^{\circ} \mathrm{C}$ ) which could not be considered as normal physiological conditions. Average core body temperature of cattle is well-known as $38.5^{\circ} \mathrm{C} \pm 0.50^{\circ} \mathrm{C}$ and even 1 or 2 degree of body temperature loss from normal state may be considered as hypothermia in homeothermic animal (Carroll et al., 2012). In most cases, such dip points in subcutaneous temperature profiles were corresponded to the lowest points in environmental temperature profiles of early morning. Therefore, it is assumed that the temporal dips under $36^{\circ} \mathrm{C}$ observed in all around a year would be due to relatively long time exposure of cattle to cold atmosphere without movement during their rest especially early in the morning and hypothesized that the circadian rhythm observed from subcutaneous temperature profiles in cattle would not be a special physiological symptoms, but be simple behavioral phenomena influenced by environmental temperature. For this reason, it should be carefully dealt with such temporal temperature dips not to be interpreted as a disease state when designing temperature-based judgment algorithm for the infectious disease. In other words, it means the subcutaneous temperature could not be used to assess the hypothermal symptoms.

From these observations, it is suggested that the usefulness of ST monitoring in cattle at field conditions as a reference for the core BT measurement is somewhat limited due to the frequent occurrence of abnormal temperature readings which could greatly lower the mean temperature and increase the temperature ranges. Early detection of body temperature above the normal range should be sensitive enough not to miss the abnormal animals and robust without frequent false alarm. These apparent outliers could be removed by an algorithm to discard the subphysiologic temperature readings (for example, cut-off set to below $36.5^{\circ} \mathrm{C}$ [19.4\% of total readings] or $37^{\circ} \mathrm{C}$ [36.4\%]). A cattle could be marked as having an alarming body temperature when a $0.8^{\circ} \mathrm{C}$ or $1^{\circ} \mathrm{C}$ deviation above baseline. For example, average of readings of previous 5 to 10 days or $5 \mathrm{~h}$ herd average of 5 days ago could be calculated with readings only above $36.5^{\circ} \mathrm{C}$ or $37^{\circ} \mathrm{C}$. If $>36.5^{\circ} \mathrm{C}$ or $>37^{\circ} \mathrm{C}$ setting is used, the mean $\pm \mathrm{SD}$ will be $37.57 \pm 0.64$ or $37.80 \pm 0.55$ which is much similar to the rectal ones (38.20 \pm 0.27 ). This algorithm could be adjusted according to the onsite results to reduce false alarm and improve the accuracy and sensitivity. This strategy was successfully applied in dairy cows at field condition for a year using reticular bolus BT monitoring system for detection of clinical mastitis and pneumonia with high specificity and sensitivity (Adams et al., 2013).

\section{Location effect for implantation of thermo-loggers}

Using implanted thermo-loggers to each steer in three different sites, lateral neck, upper scapula, and lower scapula areas, location effects were assessed for the mean ST ranges. The implantation site should be less influenced by environmental temperature and provide easy access by workers. A linear regression analysis was performed to assess co-relationship between each implantation site and influence of environmental temperature (data not shown). The upper scapula region appeared to be the least influenced by environmental temperature among the implantation sites tested. Besides, as the upper scapula area naturally forms concave pocket with enough subcutaneous space, the implantation of thermo-sensor tag could be readily accomplished with less stress to cattle (Figure 1).

In conclusion, this study provides long term subcutaneous body temperature data of cattle in field condition measured with the implanted thermo-loggers. It showed fairly stable temperature ranges with clear circadian rhythm, but with $19.4 \%$ of $<36.5^{\circ} \mathrm{C}$ or $36.4 \%$ of $<37^{\circ} \mathrm{C}$ outliers probably influenced by ambient temperature and animal stillness. Upper scapula region was the least affected site. The subcutaneous body temperature in cattle may be used as a reliable reference for the core BT to detect the infectious fever or other heat production events after proper adjustment by an algorithm to remove the outlier readings and to establish a stable baseline.

\section{CONFLICT OF INTEREST}

We certify that there is no conflict of interest with any financial organization regarding the material discussed in the manuscript.

\section{ACKNOWLEDGMENTS}

This work was supported by the IT R\&D program of MKE/KEIT (10041749, Development of livestock traceability system using noncontact sensor tags) and Advanced Production Technology Development Program, Ministry of Agriculture, Food and Rural Affairs (313002-3, Development of a u-IT based smart dairying total management system).

\section{REFERENCES}

Adams, A. E., F. J. Olea-Popelka, and I. N. Roman-Muniz. 2013. Using temperature-sensing reticular boluses to aid in the detection of production disease in dairy cows. J. Dairy Sci. 96:1549-1555.

Bewley, J. M. 2006. Automatic temperature monitoring: What are the potential benefits? http://www.uky.edu/Ag/Animal 
Sciences/dairy/dairysystems/Automatic $\% 20$ temperature $\% 20 \mathrm{~m}$ onitoring\%20potential\%20benefits.pdf Accessed July 29, 2015.

Bewley, J. M., M. E. Einstein, M. W. Grott, and M. M. Schutz. 2008. Comparison of reticular and rectal core body temperatures in lactating dairy cows. J. Dairy Sci. 91:46614672.

Bewley, J. M. and M. M. Schutz. 2010. Recent studies using a reticular bolus system for monitoring dairy cattle core body temperature. The First North American Conference on Precision Dairy Management. http://www.precisiondairy.com/ proceedings/s11bewley.pdf (Accessed July 29, 2015).

Bitman, J., A. Lefcourt, D. L. Wood, and B. Stroud. 1984. Circadian and ultradian temperature rhythms of lactating dairy cows. J. Dairy Sci. 67:1014-1023.

Brown, R. W., J. L. Thomas, H. M. Cook, J. L. Riley, and G. D. Booth. 1977. Effect of environmental temperature stress on intramammary infections of dairy cows and monitoring of body and intramammary temperatures by radiotelemetry. Am. J. Vet. Res. 38:181-187.

Carpenter, T. E., J. M. O'Brien, A. D. Hagerman, and B. A. McCarl. 2011. Epidemic and economic impacts of delayed detection of foot-and-mouth disease: A case study of a simulated outbreak in California. J. Vet. Diagn. Invest. 23:26-33.

Carroll, J. A., N. C. Burdick, C. C. Jr. Chase, S. W. Coleman, and D. E. Spiers. 2012. Influence of environmental temperature on the physiological, endocrine, and immune responses in livestock exposed to a provocative immune challenge. Domest. Anim. Endocrinol. 43:146-153.

Cilia, J., D. C. Piper, N. Upton, and J. J. Hagan. 1998. A comparison of rectal and subcutaneous body temperature measurement in the common marmoset. J. Pharmacol. Toxicol. Methods. 40:21-26.

Dalal, S. and D. S. Zhukovsky. 2006. Pathophysiology and management of fever. J. Support. Oncol. 4:9-16.

Goodwin, S. 1998. Comparison of body temperatures of goats, horses, and sheep measured with a tympanic infrared thermometer, an implantable microchip transponder, and a rectal thermometer. Contemp. Top. Lab. Anim. Sci. 37:51-55.
Huțu, I., F. Ionescu, A. Cimponeriu, and M. Chilințan. 2009. RFID technology used for identification and temperature monitoring of cattle. Lucrari Stiintifice Medicina Veterinara. 42:44-50.

Joo, Y. S., S. H. An, O. K. Kim, J. Lubroth, and J. H. Sur. 2002. Foot-and-mouth disease eradication efforts in the Republic of Korea. Can. J. Vet. Res. 66:122-124.

McLaws, M., C. Ribble, W. Martin, and J. Wilesmith. 2009. Factors associated with the early detection of foot-and-mouth disease during the 2001 epidemic in the United Kingdom. Can. Vet. J. 50:53-60.

Olsen, S. J., Y. Laosiritaworn, S. Pattanasin, P. Prapasiri, and S. F. Dowell. 2005. Poultry-handling practices during avian influenza outbreak, Thailand. Emerg. Infect. Dis. 11:16011603.

Park, J. H., K. N. Lee, Y. J. Ko, S. M. Kim, H. S. Lee, Y. K. Shin, H. J. Sohn, J. Y. Park, J. Y. Yeh, Y. H. Lee, M. J. Kim, Y. S. Joo, H. Yoon, S. S. Yoon, I. S. Cho, and B. Kim. 2013. Control of foot-and-mouth disease during 2010-2011 epidemic, South Korea. Emerg. Infect. Dis. 19:655-659.

Prendiville, D. J., J. Lowe, B. Earley, C. Spahr, and P. Kettlewell. 2002. Radiotelemetry systems for measuring body temperature. Grange Research Centre. Beef Production Series No. 57:1-14.

TekVet Health Monitoring System by TekVet Technologies Co. An external ear canal temperature monitoring system. http://www.tekvet.com/low/index.html Accessed July 29, 2015.

TempTrack by DVM Systems LLC. Rumen bolus type temperature monitoring system. http://www.dvmsystems.com/ beta7-1/ Accessed July 29, 2015.

TempVerified by FeverTags LLC. An external ear canal temperature monitoring system. http://www.fevertags.com/ Accessed July 29, 2015.

Thermo-transponder (IPPT-100 or -300) by Bio Medic Data System. http://www.bmds.com/products/transponders/iptt-300 Accessed July 29, 2015.

Won, Y. J., Y. H. Kim, Y. Lim, Y. K. Moon, and S. O. Lim. 2012. Development of livestock traceability system based on implantable RFID sensor tag with MFAN. J. Korea. Inform. Commun. Soc. 37C:1318-1327. 